\title{
Optimal contract design for dual-channel supply chains under information asymmetry
}

\author{
Honglin Yang, Erbao Cao, Kevin Jiang Lü, Guoqing Zhang
}

\begin{abstract}
This paper studies the effect of information asymmetry on revenue sharing contracts and performance in a dual-channel supply chain. Utilizing a revenue sharing contract menu design model for dual-channel supply chains under asymmetric cost information based on principal-agent theory and presenting an optimal decision model under full information for comparison with asymmetric information, an optimal contract is conceived for the manufacturer, and the impact of asymmetric information on the performance of the partners and the whole supply chain is analysed. The study determines that the direct sale price does not change while the retail sale price increases and retail demand decreases. Information asymmetry degrades the performance for the manufacturer and the whole supply chain, while retailers can benefit from asymmetric information. The performance for the manufacturer, the retailer and the whole supply chain can be improved if the condition that the retailer shares cost information can be met. Finally, numerical examples are adopted to verify our analytical findings and gain more insights into the optimal policies.
\end{abstract}

Key Words: E-commerce; supply chain management; dual-channel; information asymmetry; revenue sharing contract; game theory.

\section{Introduction}

The dual-channel structure has increased in popularity for the sale of products, combining the direct sale channel with the traditional retail channel (Cai, 2010). Over the last few years, due to the rapid growth of E-commerce and the third-party logistics enterprises, many manufacturers (such as Nike, Sony, Hewlett Packard, Dell and Lenovo) have started selling their products directly to their customers (Chen et al., 2012). The manufacturers benefit from a web-based direct channel, since it helps the manufacturers in cost saving, increasing sale revenue, expanding new market segments and avoiding retailer domination (Huang et al., 2012). The direct sale has also changed customers' purchase patterns. 
Consequently, an increasing number of manufacturers have redesigned their sale structures by adopting direct sales to satisfy different customers' purchasing preferences because the traditional retail channel was not able to meet their requirements (Cai, 2010). However, the manufacturer faces direct competition from the retailer in a dual-channel supply chain (Samar et al., 2008). Performance issues regarding the dual-channel supply chain have evoked considerable interest from both academicians and practitioners (Chiang et al., 2003). Theoretically, channel coordination can yield greater profit to partners and alleviate channel conflicts (Cachon, 2003).

However, in practice the supply chain partners' information regarding items such as raw material costs, labour costs and so on is confidential, and obtaining full information about a partner's production cost may be difficult or impossible (Laffont and Martimort, 2002; Samar, et al., 2008). The extant literature has barely addressed the issue of dual-channel supply chain coordination under asymmetric information, which motivates us to ask the following research questions: How to alleviate the channel conflict by a contract in the dual-channel supply chain? How does information asymmetry affect partners' prices, profits and overall supply chain performance? We explore the asymmetric information case and the full information case with regard to alleviating channel conflicts by considering the contract design problem. We assess the impact of asymmetric information on each partner's performance by comparing differences in profit levels under both the full information case and the asymmetric information case.

In this paper, we present two main issues associated with revenue sharing contracts to alleviate manufacturer-retailer conflicts in a dual-channel supply chain. In the first issue, a revenue sharing contract is designed in a dual-channel supply chain under asymmetric cost information conditions, based on the principal-agent model (Laffont and Martimort, 2002; Lei et al., 2012; Volodymyr et al., 2012). In the second issue, an optimal revenue sharing contract under full information conditions, based on the Stackelberg game is discussed. Under the asymmetric information case, the manufacturer offers a lump sum side payment to the retailer to alleviate channel conflict. The paper illustrates the optimum prices and production quantity in each channel under both the full information case and the asymmetric information case. The other main purpose of this study is to assess the impact of information sharing on the 
performances of the manufacturer and the retailer by comparing the profit difference between the full information case and the asymmetric information case. The study ascertains the value of information for the partners, and the conditions under which partners are willing to share confidential information.

The contributions of this study are threefold. Firstly, we model the optimum revenue sharing contract in a dual-channel supply chain under both the full information case and the asymmetric information case. Secondly, we contrast the optimal decisions of a dual-channel supply chain between the full information case and the asymmetric information case. Thirdly, we explore the impact of asymmetric cost information on the performance of a dual-channel supply chain and investigate the information value.

This study provides some interesting observations. First, the direct sale price is unchanged and independent of the retailer's cost construct, but the wholesale price increases and the price sale price does not decrease under asymmetric cost information. The information asymmetry leads to higher direct sale demand and lower retail sale demand. Second, information asymmetry is beneficial for the retailer, but imposes inefficiency on the manufacturer and the whole supply chain. Third, the performance of the dual-channel supply chain is improved if the retailer's cost information is shared and the dual-channel supply chain reaches coordination. The retailer is willing to share its cost information if the lump sum side payment that the manufacturer offers can make up the retailer's reduced profit due to sharing this information.

The remainder of this paper is organized as follows. Section 2 introduces the published relative literature. In Section 3, we briefly describe the model set up, including the optimal contracts under asymmetric information based on the principal-agent theory in Section 3.1, and the optimal contract under full information in Section 3.2. Section 4 discusses the value of information sharing. In Section 5, the numerical examples with analytical results are presented. We conclude the results and limitations in Section 6.

\section{Related Work}

This paper addresses the intersection of the dual-channel supply chain and information 
asymmetry. The related literatures, including those on dual-channel supply chain, revenue sharing contract and information asymmetry are reviewed and analysed in this section.

Channel competition and the coordination of the dual-channel supply chain have received increasing attention in the past few years. However, the competition of a dual-channel may lead to channel conflicts and inefficiency. The determination and adoption of effective methods to enhance the performance of the dual-channel supply chain have become interesting topics for both academicians and practitioners (Chiang et al., 2003). Theoretically, channel coordination can yield greater profits to partners and alleviate channel conflicts (Cachon, 2003). Studies of channel structures have experienced growing popularity in recent years. Chiang et al. (2003) finds that retailers also benefit from the direct channel by decreasing a wholesale price when considering a price-setting game between retail and direct channels. Swaminathan and Tayur (2003) reviews relevant analytical models and discusses the strengths and limitations of different models in dual supply chains. Yao and Liu (2003) examine the customer diffusion between two channels and point out that both channels have stable demands under certain conditions. Dumrongsiri et al. (2008) studies the pricing and service quality models in a dual-channel supply chain. Yan (2008) studies the optimal prices decision model based on game theory in dual-channel supply chains. Cai (2010) discusses the influences of different channel structures and channel coordination on the profits of supply chain partners and the system as a whole. Hua et al. (2010) compares the optimal delivery lead time and prices under a centralized decision case and a decentralized decision case in a dual-channel supply chain. Dan et al. (2012) compares the optimal price between centralized decision making and decentralized decision making, and presents a coordination strategy. However, studies on contracts that provide insights on how to coordinate a dual-channel supply chain are limited. Chen et al. (2012) investigates a manufacturer's pricing strategies in a dual-channel supply chain, and finds that a wholesale price contract and a direct channel price can coordinate the dual-channel supply channel.

Over the last two decades, researches on supply chain coordination have gained much attention from academicians and practitioners. Contracts with various coordination mechanisms are widely used in supply chain coordination (Cachon, 2003). A revenue sharing contract is beneficial to manufacturers, retailers and other participants in a supply chain (Lei 
et al., 2012). Wang et al. (2004) studies the channel performance of supply chains under consignment contract with revenue sharing, finding that the performance of the overall channel as well as individual firms critically depends on demand price elasticity and the retailer's share of channel cost, and that a decentralized supply chain cannot be coordinated. $\mathrm{Li}$ and Hua (2008) extend the work of Wang et al. (2004) and provide a cooperative game model that implements revenue sharing between the manufacturer and the retailer to ensure cooperation. Cachon and Lariviere (2005) study revenue-sharing contracts in a two-echelon distribution channel with competing retailers, finding that in an extended setting where the market demand is both stochastic and retail price sensitive, only one revenue sharing contract can coordinate the channel. Yao et al. (2008) investigate a revenue-sharing contract for coordinating a supply chain with one manufacturer and two competing retailers, assuming demands from these two competing retailers to be independent and that all parties know their demand distributions at the beginning of the season, but they do not consider asymmetric information. Yao et al. suggest that the revenue-sharing contract has better performance than a wholesale price-only contract. None of these studies consider the revenue sharing contract for coordinating the dual-channel supply chain. In this paper, we consider not only pricing decisions but also the issue of coordination in a dual-channel supply chain under the asymmetric information case and the full information case.

Information asymmetry in the supply chain is another aspect closely related to this study. Information asymmetry has attracted increasing attention in the past few years (Laffont and Martimort, 2002; Lei et al., 2012; Volodymyr et al., 2012). Information asymmetry is very common in the supply chain and has received substantial attention recently (Laffont and Martimort, 2002; Samar, et al., 2008). Laffont and Martimort (2002) study the incentives theory based on the principal-agent model. Charles et al. (2004) study the wholesale price contracts design problem under cost information asymmetry and make a comparison with full information. Samar et al. (2008) examine the information sharing of value-adding retailers in a dual-channel hi-tech supply chain, based on the Stackelberg game. Volodymyr et al. (2012) investigate a buyback contract design problem under demand information asymmetry. Cakanyildirim et al. (2012) study the contract design and coordination problems under asymmetric production of cost information in a one-supplier and one-retailer supply chain 
where the supplier has type-dependent reservation profit.

To the best of our knowledge, the revenue sharing contract design problem in a dual-channel supply chain has not so far been considered. There are several differences between our model and the above papers. Firstly, we consider a revenue sharing contract to alleviate the channel conflict under the cases of asymmetric information and full information. Furthermore, unlike Cai (2010) and other dual-channel pricing and coordination research, we investigate the influences of information asymmetry on prices, production quantities and profits.

\section{The Model}

We examine a dual-channel supply chain consisting of a direct sale channel in addition to the traditional retail channel, which is composed of a manufacturer and a retailer. The manufacturer may sell the products to the retailer, and may also sell the products to end customers directly. Thus, in a way, the retailer and the manufacturer compete on prices in the end customer market.

The retail price and direct sale price to the end customers are $p_{r}$ and $p_{d}$ respectively. This price competition between the direct channel and the retailer can be modelled as differentiated Bertrand competition (Chen et al., 2012). We assume that there is only one product for sale in the end market (Huang et al., 2012). The retailer is charged the wholesale price $w$ by the manufacturer. Let $c_{m}$ equal the unit cost of manufacturer in direct channel and $c_{r}$ the unit cost of retailer in the retail channel. The manufacturer and retailer are considered as playing a Stachelberg game, where the manufacturer acts as a leader and a retailer as a follower (Huang et al., 2012). We considered two cases regarding the retailer's costs: the full information case, where the manufacturer knows exactly $c_{r}$; and the asymmetric information case, where the manufacturer only knows that there are two types of retail selling costs: the high cost $\overline{c_{r}}$ with probability $\theta$ and the low $\operatorname{cost} c_{r}$ with probability $1-\theta$. To facilitate the analysis, following Yue and Liu (2006), Chen et al., (2012), Huang et al, (2012), we assume that the end market 
demand for the two channels is formulated as follows:

$$
\begin{aligned}
& D_{r}=(1-\rho) a-\alpha_{1} p_{r}+\beta p_{d}, \\
& D_{d}=\rho a-\alpha_{2} p_{d}+\beta p_{r},
\end{aligned}
$$

Where $D_{r}$ and $D_{d}$ are the demands in the retail channel and the direct channel respectively. Eq. (1) and Eq. (2) indicate that the each channel's demand, linearly depends on the retail price $p_{r}$ and the direct sale price $p_{d}, a$ is the base potential demand, capturing the effects of all variables not considered in our model. The demand share of the direct channel is $\rho$ and the rest $1-\rho$ represents the demand share in the retail channel when the products are free of charge, $\rho$ reflects the end customer's preference for the direct channel (Chen et al., 2012). $\alpha_{1}$ and $\alpha_{2}$ respectively represent the coefficients of self-price sensitiveness of the demand $D_{r}$ and $D_{d}$, which means that this is the reduction from the base demand per unit self-price increase. $\beta$ is the coefficient of cross-price sensitivity, which reflects the degree of competition between the two channels and the migration rate if customers perceive that there is a difference between the retail price $p_{r}$ and the direct sale price $p_{d}$ (Huang et al., 2012). We assume that $\alpha_{i}>\beta, p_{d} \geq w$. Otherwise, the retailer will prefer to obtain products from the direct channel. In order to coordinate the dual-channel supply chain, we primarily investigate a revenue sharing contract $(w, \phi)$ in which the retailer obtains the product from the manufacturer with wholesale price $w$ and shares $1-\phi$ percentage of its revenue with the manufacturer.

With the above assumptions and notations, the retailer's profit function is determined by

$$
\pi_{r}=\left(\phi \mathrm{p}_{r}-\mathrm{W}_{-} \mathrm{c}_{r}\right) \mathrm{D}_{r},
$$

The manufacturer's profit function is determined by

$$
\pi_{m}=\left((1-\phi) \mathrm{p}_{r}+\mathrm{w}\right) D_{r}+\left(\mathrm{p}_{d}-\mathrm{c}_{m}\right) \mathrm{D}_{d},
$$

And the dual-channel supply chain's joint profit function is

$$
\pi=\pi_{r}+\pi_{m}=\left(\mathrm{p}_{r}-\mathrm{c}_{r}\right) \mathrm{D}_{r}+\left(\mathrm{p}_{d}-\mathrm{c}_{m}\right) \mathrm{D}_{d},
$$




\subsection{The asymmetric information case}

In this study, it is assumed that the retailer has its own private information about its cost $c_{r}$ and has two cost states: a low state $\underline{c_{r}}$ and a high state $\overline{c_{r}}$. The retailer knows which of the two cost states will occur. Meanwhile the manufacturer has only a subjective assessment about the likelihood of the two cost states. The manufacturer only knows that the high cost $\overline{c_{r}}$ with probability $\theta$ and the low $\operatorname{cost} c_{r}$ with probability $1-\theta$. Although the assumption regarding the two cost states is a simplification of the reality, these two-costs are sufficient to capture the major effect of information on contracting and competition. This type of asymmetric information has been commonly adopted in supply chain contracting or information screening literatures (for example, Laffont and Martimort, 2002; and Cakanyildirim et al., 2012) due to its parsimony and tractability for analysis. In this study, the manufacturer is an uninformed party that acts as a principal, and the retailer is treated as an agent that holds private information about its costs. We consider the revenue sharing contract design problem under asymmetric cost information. The goal of the manufacturer is to design a menu of revenue sharing contracts so as to maximize its expected profit based on the principal-agent model ( Laffont and Martimort, 2002; Lei et al., 2012; Volodymyr et al., 2012 ).

The decision procedure is as follows: (1) the retailer's cost state is observed by the manufacturer; (2) the manufacturer offers a menu of revenue sharing contracts to the retailer $\{(\bar{w}, \bar{\phi}),(\underline{w}, \underline{\phi})\} ;(3)$ the retailer takes one of the contracts from the menu or leaves it; (4) if the retailer chooses to take one of the contracts, the manufacturer must sell $\overline{D_{r}}\left(\underline{D_{r}}\right)$ units of product to the retailer at the wholesale price $\bar{w}(\underline{w})$, and gains $1-\phi$ of sale revenue from the retailer. The manufacturer's problem is how to set the menu of revenue sharing contracts to maximize its expected profit. Without loss of generality, we suppose that the retailer's reservation profit is 0 . Under asymmetric cost information, the manufacturer's decision problem is presented as follows:

$$
\max _{\{(\bar{w}, \bar{\phi}),(\underline{w}, \underline{\phi},\})} \pi_{m}=\theta\left[\left((1-\bar{\phi}) \overline{p_{r}}+\bar{w}\right) \overline{D_{r}}+\left(\overline{p_{d}}-c_{m}\right) \overline{D_{d}}\right]+(1-\theta)\left[\left((1-\underline{\phi}) \underline{p_{r}}+\underline{w}\right) \underline{D_{r}}+\left(\underline{p_{d}}-c_{m}\right) \underline{D_{d}}\right],
$$




$$
\begin{array}{ll}
\text { s.t. } \quad & \left(\bar{\phi} \overline{p_{r}}-\bar{w}-\overline{c_{r}}\right) \overline{D_{r}} \geq 0, \\
& \left(\underline{\phi} \underline{p_{r}}-\underline{w}-\underline{c_{r}}\right) \underline{D_{r}} \geq 0, \\
& \left(\bar{\phi} \overline{p_{r}}-\bar{w}-\overline{c_{r}}\right) \overline{D_{r}} \geq\left(\underline{\phi} \underline{p_{r}}-\underline{w}-\overline{c_{r}}\right) \underline{D_{r}}, \\
& \left(\underline{\phi} \underline{p_{r}}-\underline{w}-\underline{c_{r}}\right) \underline{D_{r}} \geq\left(\bar{\phi} \overline{p_{r}}-\bar{w}-\underline{c_{r}}\right) \overline{D_{r},}
\end{array}
$$

Eq. (7) and Eq. (8) are individual rationality constraints, they assure the retailer will join the dual-channel supply chain because its profit exceeds the reservation profit. Eq. (9) and Eq. (10) are incentive compatibility constraints, they force each type of the retailer to truly announce their type.

From Eq. (7) and Eq. (10), Eq. (8) is always strictly satisfied. Thus we have

$$
\begin{gathered}
\left(\bar{\phi} \overline{p_{r}}-\bar{w}-\overline{c_{r}}\right) \overline{D_{r}}=0, \\
\left(\underline{\phi} \underline{p_{r}}-\underline{w}-\underline{c_{r}}\right) \underline{D_{r}}=\left(\bar{\phi} \overline{p_{r}}-\bar{w}-\underline{c_{r}}\right) \overline{D_{r}},
\end{gathered}
$$

Substituting (11) into (12), we have

$$
\left(\underline{\phi} \underline{p_{r}}-\underline{w}-\underline{c_{r}}\right) \underline{D_{r}}=\left(\bar{\phi} \overline{p_{r}}-\bar{w}-\underline{c_{r}}\right) \overline{D_{r}}-\left(\bar{\phi} \overline{p_{r}}-\bar{w}-\overline{c_{r}}\right) \overline{D_{r}}=\left(\overline{c_{r}}-\underline{c_{r}}\right) \overline{D_{r}},
$$

Substituting Eq. (11) and Eq. (13) into Eq. (6), the manufacturer's decision problem can be transformed to:

$$
\begin{aligned}
& \max _{\{(\bar{w}, \bar{\phi}),(\underline{w}, \underline{\phi})\}} \pi_{m}=\theta\left[\left((1-\bar{\phi}) \overline{p_{r}}+\bar{w}\right) \overline{D_{r}}+\left(\overline{p_{d}}-c_{m}\right) \overline{D_{d}}\right]+(1-\theta)\left[\left((1-\underline{\phi}) \underline{p_{r}}+\underline{w}\right) \underline{D_{r}}+\left(\underline{p_{d}}-c_{m}\right) \underline{D_{d}}\right] \\
&= \theta\left[\left(\overline{p_{r}}-\overline{c_{r}}\right) \overline{D_{r}}+\left(\overline{p_{d}}-c_{m}\right) \overline{D_{d}}\right]+(1-\theta)\left[\left(\underline{p_{r}}-\underline{c_{r}}\right) \underline{D_{r}}-\left(\overline{c_{r}}-\underline{c_{r}}\right) \overline{D_{r}}+\left(\underline{p_{d}}-c_{m}\right) \underline{D_{d}}\right] \\
&= \theta\left[\left(\overline{p_{r}}-\overline{c_{r}}\right)\left[(1-\rho) a-\alpha_{1} \overline{p_{r}}+\beta \overline{p_{d}}\right]+\left(\overline{p_{d}}-\mathrm{c}_{m}\right)\left(\rho a-\alpha_{2} \overline{p_{d}}+\beta \overline{p_{r}}\right)\right]+ \\
&(1-\theta)\left[\left(\underline{p_{r}}-\underline{c_{r}}\right)\left((1-\rho) a-\alpha_{1} \underline{p_{r}}+\beta \underline{p_{d}}\right)-\left(\overline{c_{r}}-\underline{c_{r}}\right)\left[(1-\rho) a-\alpha_{1} \overline{p_{r}}+\overline{\beta p_{d}}\right]+\left(\underline{p_{d}}-\mathrm{c}_{m}\right)\left(\rho a-\alpha_{2} \underline{p_{d}}+\beta \underline{p_{r}}\right)\right],
\end{aligned}
$$

From Eq. (11) and Eq. (13), we can know that $\bar{w}=\bar{\phi} \overline{p_{r}}-\overline{c_{r}}$ and $\underline{w}=\underline{\phi} \underline{p_{r}}-\underline{c_{r}}-\left(\overline{c_{r}}-\underline{c_{r}}\right) \frac{\overline{D_{r}}}{\underline{D_{r}}}$. From the first order optimal condition of Eq. (14), when the retailer's cost is high we derive the optimal decisions as follows: 


$$
\left\{\begin{array}{c}
\overline{p_{r}}=\frac{a\left[-\beta \rho+(-1+\rho) \alpha_{2}\right]}{2\left(\beta^{2}-\alpha_{1} \alpha_{2}\right)}+\frac{\overline{c_{r}}+(-1+\theta) \underline{c_{r}}}{2 \theta} \\
\overline{p_{d}}=\frac{1}{2}\left[c_{m}+\frac{\left.a \beta(-1+\rho)-a \rho \alpha_{1}\right]}{\beta^{2}-\alpha_{1} \alpha_{2}}\right] \\
\bar{w}=\frac{1}{2}\left[\left(-2+\frac{\bar{\phi}}{\theta}\right) \overline{c_{r}}+\frac{-a \beta \rho+(-1+\rho) a \alpha_{2}}{\beta^{2}-\alpha_{1} \alpha_{2}} \bar{\phi}+\frac{(-1+\theta) \underline{c_{r}}}{\theta} \bar{\phi}\right] \\
\overline{D_{r}}=\frac{a \theta(1-\rho)+\beta \theta \mathrm{c}_{m}-\alpha_{1}\left[\overline{c_{r}}+(-1+\theta) \underline{c_{r}}\right]}{2 \theta} \\
\overline{D_{d}}=\frac{a \rho-\alpha_{2} c_{m}}{2}+\frac{\beta \overline{c_{r}}+\beta(-1+\theta) \underline{c_{r}}}{2 \theta} \\
\bar{D}=\frac{\left(\beta-\alpha_{1}\right)\left[\overline{c_{r}}+(-1+\theta) \underline{c_{r}}\right]}{2 \theta}+\frac{a+c_{m}\left(\beta-\alpha_{2}\right)}{2}
\end{array}\right.
$$

and the revenue share $\phi$ should satisfy the following condition

$$
0<\bar{\phi}<\frac{\left(c_{m}+2 \overline{c_{r}}\right)\left(\beta^{2}-\alpha_{1} \alpha_{2}\right)+a \beta(-1+\rho)-a \rho \alpha_{1}}{\left(\beta^{2}-\alpha_{1} \alpha_{2}\right) \frac{\overline{c_{r}}+(-1+\theta) \underline{c_{r}}}{\theta}+a \rho\left(\alpha_{2}-\beta\right)-a \alpha_{2}} .
$$

When the retailer's cost is low, we also obtain the optimal decisions as follows:

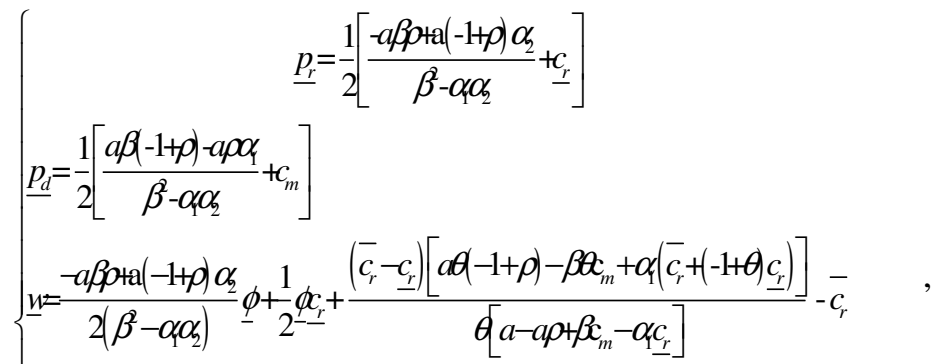

$$
\begin{aligned}
& \underline{D_{r}}=\frac{1}{2}\left[a-a \rho+\beta c_{m}-\alpha_{\underline{r}} c_{r}\right] \\
& \underline{D_{d}}=\frac{1}{2}\left[\rho a-\alpha_{2} c_{m}+\beta c_{r}\right] \\
& \underline{D}=\frac{1}{2}\left[a+c_{m}\left(\beta-\alpha_{2}\right)+\left(\beta-\alpha_{1}\right) c_{r}\right]
\end{aligned}
$$

and the revenue share $\phi$ must satisfy the condition $0<\underline{\phi}<\frac{\frac{a \beta(-1+\rho)-a \rho \alpha_{1}}{\beta^{2}-\alpha_{1} \alpha_{2}}+c_{m}+2 \underline{c_{r}}-\frac{\left(\overline{c_{r}}-\underline{c_{r}}\right)\left[a \theta(-1+\rho)-\beta \theta c_{m}+\alpha_{1}\left(\overline{c_{r}}+(-1+\theta) \underline{c_{r}}\right)\right]}{\theta\left[a-a \rho+\beta \mathrm{c}_{m}-\alpha_{1} \underline{c_{r}}\right]}}{\frac{-a \beta \rho+\mathrm{a}(-1+\rho) \alpha_{2}}{\beta^{2}-\alpha_{1} \alpha_{2}}+\underline{c_{r}}}$. 


\subsection{The full information case}

Under full information, the manufacturer and retailer have full information about the dual-channel supply chain. Therefore, the incentive compatibility constraints Eq. (9) and Eq. (10) no longer work. Moreover, the individual rationality constraints Eq. (7) and Eq. (8) must both be binding at the optimal solution of the manufacturer's problem. Thus the manufacturer's problem can be transformed to the following equations:

$$
\begin{aligned}
\max _{\{(\bar{w}, \bar{\phi}),(w, \underline{\underline{\phi}} \hat{\underline{\phi}}\}} \pi_{m}= & \oint\left[\left((1-\bar{\phi}) \overline{p_{r}}+\bar{w}\right) \overline{D_{r}}+\left(\overline{p_{d}}-c_{m}\right) \overline{D_{d}}\right]+(1-\theta)\left[\left((1-\underline{\phi}) \underline{p_{r}}+\underline{w}\right) \underline{D_{r}}+\left(\underline{p_{d}-c_{m}}\right) \underline{D_{d}}\right], \\
\text { s.t. } \quad & \left(\bar{\phi} \overline{p_{r}}-\bar{w}-\overline{c_{r}}\right) \overline{D_{r}}=0, \\
& \left(\underline{\phi} \underline{p_{r}}-\underline{w}-\underline{c_{r}}\right) \underline{D_{r}}=0
\end{aligned}
$$

So we can derive the optimal decisions under retailer's high cost state:

$$
\left\{\begin{array}{c}
\overline{p_{r}^{*}}=\frac{a \beta \rho+a(1-\rho) \alpha_{2}+\overline{c_{r}}\left(\alpha_{1} \alpha_{2}-\beta^{2}\right)}{2\left(\alpha_{1} \alpha_{2}-\beta^{2}\right)} \\
\overline{p_{d}^{*}}=\frac{1}{2}\left[c_{m}+\frac{a \beta(-1+\rho)-a \rho \alpha_{1}}{\beta^{2}-\alpha_{1} \alpha_{2}}\right] \\
\overline{w^{*}}=\frac{1}{2}\left[(-2+\bar{\phi}) \overline{c_{r}}-\frac{a \bar{\phi}\left(\beta \rho+(1-\rho) \alpha_{2}\right)}{\beta^{2}-\alpha_{1} \alpha_{2}}\right] \\
\overline{D_{r}^{*}}=\frac{1}{2}\left[a-a \rho+\beta c_{m}-\overline{c_{r}} \alpha_{1}\right] \\
\overline{D_{d}^{*}}=\frac{1}{2}\left[a \rho+\beta \overline{c_{r}}-c_{m} \alpha_{2}\right] \\
\overline{D^{*}}=\frac{1}{2}\left[a+\overline{c_{r}}\left(\beta-\alpha_{1}\right)+c_{m}\left(\beta-\alpha_{2}\right)\right] \\
\overline{\pi_{r}^{*}}=0 \\
\overline{\pi_{m}^{*}=}=\frac{\left(a \rho+\beta \overline{c_{r}}\right)\left[2 a \beta(-1+\rho)-2 \beta^{2} c_{m}+\left(-a \rho+\beta \overline{c_{r}}\right) \alpha_{1}\right]-\alpha_{2}\left[\left(a \rho-a+\overline{c_{r}} \alpha_{1}\right)^{2}-2\left(a \rho+\beta \overline{c_{r}}\right) c_{m} \alpha_{1}-\beta^{2} c_{m}^{2}\right]-c_{m}^{2} \alpha_{1} \alpha_{2}^{2},}{4\left(\beta^{2}-\alpha_{1} \alpha_{2}\right)},
\end{array}\right.
$$

the optimal decisions under retailer's low cost state are as follows: 


$$
\begin{aligned}
& \underline{p_{r}^{*}}=\frac{1}{2}\left[\frac{a \beta \rho+a(1-\rho) \alpha_{2}}{\alpha_{1} \alpha_{2}-\beta^{2}}+c_{r}\right] \\
& \underline{p_{d}^{*}}=\frac{1}{2}\left[c_{m}+\frac{a \beta(-1+\rho)-a \rho \alpha_{1}}{\beta^{2}-\alpha_{1} \alpha_{2}}\right] \\
& \underline{w}=\frac{1}{2} \underline{\underline{\phi}}\left[\frac{a \beta \rho+a(1-\rho) \alpha_{2}}{\alpha_{1} \alpha_{2}-\beta^{2}}+\underline{c_{r}}\right] \\
& \underline{D_{r}^{*}}=\frac{1}{2}\left[a-a \rho+\beta c_{m}-\alpha_{1} \underline{c}_{r}\right] \\
& \underline{D_{d}^{*}}=\frac{1}{2}\left[a \rho+\beta c_{\underline{r}}-c_{m} \alpha_{2}\right] \\
& \underline{D}=\frac{1}{2}\left[a+c_{m}\left(\beta-\alpha_{2}\right)+\left(\beta-\alpha_{1}\right) \underline{c}_{r}\right] \\
& \underline{\pi_{m}^{*}}=\frac{\left(a \rho+\beta \underline{c_{r}}\right)\left[2 a \beta(-1+\rho)-2 \beta^{2} c_{m}+\left(-a \rho+\beta c_{r}\right) \alpha_{1}\right]-\alpha_{2}\left[\left(a \rho-a+c_{r} \alpha_{1}\right)^{2}-2\left(a \rho+\beta c_{r}\right) c_{m} \alpha_{1}-\beta^{2} c_{m}^{2}\right]-c_{m}^{2} \alpha_{1} \alpha_{2}^{2}}{4\left(\beta^{2}-\alpha_{1} \alpha_{2}\right)},
\end{aligned}
$$

By comparing the decisions under asymmetric information and full information, we can conclude the following.

\section{Theorem 1.}

(i) Comparing the optimal decisions when the retailer is in a high cost state and has full information, we have:

$\overline{w^{*}}<\bar{w} ; \overline{p_{r}^{*}}<\overline{p_{r}}, \overline{p_{d}^{*}}=\overline{p_{d}} ; \overline{D_{r}^{*}}>\overline{D_{r}}, \overline{D_{d}{ }^{*}}<\overline{D_{d}}, \overline{D^{*}}>\bar{D}$.

(ii) Comparing the optimal decisions when the retailer is in a low cost state and has full information, we have:

$\underline{w^{*}}>\underline{w} ; \underline{p_{r}^{*}}=\underline{p_{r}}, \underline{p_{d}^{*}}=\underline{p_{d}} ; \underline{D_{r}^{*}}=\underline{D_{r}}, \underline{D_{d}^{*}}=\underline{D_{d}}, \underline{D^{*}}=\underline{D}$.

Theorem 1 characterizes the impact of asymmetric cost information on the decisions of each channel partner in a dual-channel supply chain. We found that the direct sale price is unchanged and is independent of the retailer's cost. Information asymmetry leads to higher wholesale price and a higher retail sale price, lower retail sale demand and total demand but higher direct sale demand when the retailer is in a high cost state. Moreover, when the retailer is in a low cost state, only the wholesale price is influenced by the absence of information, other variables remain steady, as under symmetric information; the influence of asymmetric information is limited.

\section{Theorem 2.}

(i) When the retailer is in a high cost state, the following results hold: 
$\overline{\pi_{r}^{*}}=\overline{\pi_{r}}, \quad \overline{\pi_{m}^{*}}>\overline{\pi_{m}}, \quad \overline{\pi_{r}^{*}}+\overline{\pi_{m}^{*}}>\overline{\pi_{r}}+\overline{\pi_{m}}$

(ii) When the retailer is in a low cost state, the following results hold:

$\underline{\pi_{r}^{*}}<\underline{\pi_{r}}, \underline{\pi_{m}^{*}}>\underline{\pi_{m}}, \underline{\pi_{r}^{*}}+\underline{\pi_{m}^{*}}=\underline{\pi_{r}}+\underline{\pi_{m}}$.

The proof is straightforward and demonstrated by substituting the relevant optimal decisions into the manufacturer's profit function and retailer's profit function.

Theorem 2 quantifies the impact of asymmetric cost information on the performances of partners in a dual-channel supply chain. From Theorem 1 and Theorem 2, we know that when the retailer's cost is in a low state, the asymmetric information decreases the wholesale price, while other variables are steady, which leads to a decrease in the manufacturer's profit, while the retailer gains a greater profit from the information advantage. When the retailer is in a high cost state, the manufacturer also suffers a reduction in profit, while the retailer achieves its reservation profit. So the asymmetric information is always beneficial to the retailer, while potentially harmful to the manufacturer's profit.

Therefore, the manufacturer and the whole supply chain will always gain at the cost of the retailer when the retailer's cost information is shared. The manufacturer's profit increment is not lower than the retailer's reduction in profit. Therefore, the retailer is willing to share its information if the manufacturer can offer a lump sum side payment to the retailer to make up for the retailer's profit loss. Theorem 2 illustrates that the dual-channel supply chain can achieve the coordination under asymmetric information if the manufacturer is willing to offer a lump sum side payment to the retailer in order to make up the retailer's profit loss. The side payment the manufacturer offers should not be less than the profit difference between the asymmetric information case and the full information case.

\section{The value of information sharing}

Theorem 2 shows that the manufacturer may suffer profit loss when the retailer does not share cost information, while the retailer benefits from the asymmetric information and the retailer's profit is not less than that under full information. The value of information sharing to the manufacturer (or retailer) is the profit difference from the asymmetric information case 
to the full information case. The value of information sharing for an entity of the dual-channel supply chain is the difference between its optimal profits under full information and those under asymmetric information. What is the maximum amount the manufacturer is willing to spend for the retailer's cost information? What is the condition that governs the retailer's motivation to share its private cost information (or not)? And what is the value of information sharing for the dual-channel supply chain?

From Theorem 2, we know that the manufacturer's profit will always increase from the asymmetric information case to the full information case. The maximum amount the manufacturer is willing to spend for the retailer's cost information should not more than the incremental profit that is obtained from the asymmetric information case to the full information case. The retailer may share its cost information if the payment that is paid by the manufacturer can make up for the retailer's lost profit. The difference in the profit of the partners and that of the whole dual-channel supply chain between the full information case and the asymmetric information case can be thought of as the value of information sharing to the partners and the whole dual-channel supply chain. In order to quantify the value of information sharing to the manufacturer, we investigate the value of the asymmetric cost information from both the partners' and whole system's points of view. Now let us look at Theorem 3.

\section{Theorem 3.}

When the retailer's cost information is shared, the value of information sharing to the manufacturer $\Delta \pi_{\mathrm{m}}$ is positive. And the amount of increase in the manufacturer's profit $\Delta \pi_{\mathrm{m}}$ is given by:

$$
\Delta \pi_{\mathrm{m}}=(1-\theta)\left(\overline{c_{r}}-\underline{c_{r}}\right) \overline{D_{r}}+\frac{(1-\theta)^{2} \alpha_{1}\left(\overline{c_{r}}-\underline{c_{r}}\right)^{2}}{4 \theta}>0,
$$

From Theorem 2, we know that the manufacturer obtains all the profit of the dual-channel supply chain under full information, while the retailer only obtains the reservation profit. Under asymmetric cost information, when the retailer is in a high cost state,

the manufacturer's profit may decrease $\frac{(1-\theta)^{2} \alpha_{1}\left(\overline{c_{r}}-\underline{c_{r}}\right)^{2}}{4 \theta^{2}}$ due to the asymmetric cost 
information, when the retailer is in a low cost state, the manufacturer has to pay the information rent $\left(\overline{c_{r}}-\underline{c_{r}}\right) \overline{D_{r}}$ to the retailer to compensate any loss due to the sharing of the cost information. So the value of information sharing to the manufacturer is $(1-\theta)\left(\overline{c_{r}}-\underline{c_{r}}\right) \overline{D_{r}}+\frac{(1-\theta)^{2} \alpha_{1}\left(\overline{c_{r}}-\underline{c_{r}}\right)^{2}}{4 \theta} . \Delta \pi_{\mathrm{m}}$ is the maximum amount that the manufacturer will be willing to spend for the information sharing, and the manufacturer offers $\Delta \pi_{\mathrm{m}}$ to retailer by the lump sum side payment.

From the retailer's point of view, it is possible that the retailer will stand to lose if the retailer shares its cost information with the manufacturer. The threshold value $\left(\overline{c_{r}}-\underline{c_{r}}\right) \overline{D_{r}}$ is the condition that induces the retailer to share its cost information. In addition, the profit difference for a retailer with a high cost state is 0 from the full information case to the asymmetric information case, in that the retailer earns only the reservation profit (0) under the high cost state and the full information case. However, the profit decreases $\left(\overline{c_{r}}-\underline{c_{r}}\right) \overline{D_{r}}$ for a retailer with a low cost state from the asymmetric information case to the full information case, so the value of information sharing for the retailer with a low cost state is $-\left(\overline{c_{r}}-\underline{c_{r}}\right) \overline{D_{r}}$. This is because that retailer is dominated by the manufacturer, and the manufacturer maximizes its own profit via the information policy at the cost of a reduction in the retailer's profit. This loss in profit $\left(\overline{c_{r}}-\underline{c_{r}}\right) \overline{D_{r}}$ can be compensated as an incentive from the manufacturer if the manufacturer pursues the retailer to share its cost information. Consequently, the value of information sharing for the entity of the dual-channel supply chain is $\frac{(1-\theta)^{2} \alpha_{1}\left(\overline{c_{r}}-\underline{c_{r}}\right)^{2}}{4 \theta}$.

Eq. (22) implies that the value of information sharing for the manufacturer increases with the range of the retailer's cost, i.e. with $\overline{c_{r}}-\underline{c_{r}}$, and with the probability of retailer high $\operatorname{cost} \theta$ and the price sensitivity of retail sale demand, i.e., with $\alpha_{1}$. This means that the less certain the manufacturer is about the retailer's cost information, the higher is the value of information sharing for the manufacturer. An important managerial insight obtained here is that the 
manufacturer should decrease the predicted range about the retailer's cost or improve the predicted precision accuracy about the retailer's cost by using information technology and an incentive mechanism to collect the retailer's cost information.

Eq. (22) reveals some important findings. The advantages of information sharing to the manufacturer and the whole dual-channel supply chain are intuitive because information sharing helps the manufacturer make a better whole price decision and revenue sharing contract decision, which improves the performances of the partners and the whole system. From the above analysis, it can be seen that the manufacturer and the whole supply chain can always increase profit if the retailer's cost information is shared. If the payment charged to the

manufacturer by the retailer is more than $\left(\overline{c_{r}}-\underline{c_{r}}\right) \overline{D_{r}}$, the retailer is willing to share its cost information in that its profit is higher than that in the case that the information is not shared. An important managerial insight obtained here is that the manufacturer should actively pursue the retailer to share its cost information and pay the retailer as incentive for the information sharing. Thus sharing information can increase the profits of partners and the whole supply chain system, and enable both the manufacturer and the retailer to achieve a double win. Therefore, from the position of the manufacturer as a leader in dual-channel supply chain, sharing information by advanced information technology and management approaches in a dual-channel supply chain also can improve the profit of the manufacturer and the whole supply chain system.

\section{Numerical examples}

In this section, we use numerical examples to verify our analytical findings and gain further insights into optimal policies, especially for the varied exogenous parameters. We create an example where $\rho=0.4, a=200, \alpha_{1}=0.8, \alpha_{2}=1.2, \beta=0.6 . c_{m}=15, \underline{c_{r}}=15, \overline{c_{r}}=20$, $\theta=0.6$. We derive the revenue share $\bar{\phi}=0.4$ and $\underline{\phi}=0.6$. Table 1 shows the results for both the full information case and the asymmetric information case in a dual-channel supply chain. From Table 1, we know that the direct sale price (120.833) is unchanged and independent of the retailer's cost construct. When the retailer is in a high cost state, the 
retailer price and wholesale price under the asymmetric information case are higher than those of the full information case. Moreover, when the retailer is in a low cost state, only the wholesale price is influenced by the unshared information, while the asymmetric information does not change direct demand, retailer demand or retailer price because these decision variables are independent of the probability of the cost state $(\theta)$. From Table 1, we know that the profits of the manufacturer and the whole supply chain in the asymmetric case are lower than those in the full information case, while the retailer gains more profit in the asymmetric information case due to the advantage of information. We find that asymmetry of information is always beneficial to the retailer due to the advantage afforded them by their having full information, while decreasing the manufacturer's profit and leading to a reduction in profit to the whole dual-channel supply chain.

Table 1. Comparisons of optimal policies under full information and asymmetric information case

\begin{tabular}{|c|c|c|c|c|c|c|c|c|c|c|c|}
\hline \multicolumn{2}{|l|}{ Models } & $c_{r}$ & $p_{r}$ & $p_{d}$ & $w$ & $D_{r}$ & $D_{d}$ & $D$ & $\pi_{r}$ & $\pi_{m}$ & $\pi_{r}+\pi_{m}$ \\
\hline \multirow{2}{*}{$\begin{array}{c}\text { Full } \\
\text { information }\end{array}$} & $\begin{array}{l}\text { High } \\
\text { cost }\end{array}$ & $\bar{c}_{r}=0$ & 170 & 120.833 & 48 & 56.5 & 37 & 93.5 & 0 & 12390.8 & 12390.8 \\
\hline & $\begin{array}{l}\text { Low } \\
\text { cost }\end{array}$ & $\underline{c}_{r}=15$ & 167.5 & 120.833 & 85.5 & 58.5 & 35.5 & 94 & 0 & 12678.3 & 12678.3 \\
\hline \multirow{2}{*}{$\begin{array}{l}\text { Asymmetric } \\
\text { information }\end{array}$} & $\begin{array}{l}\text { High } \\
\text { cost }\end{array}$ & $\bar{c}_{r}=20$ & 171.667 & 120.833 & 48.667 & 55.1667 & 38 & 93.1667 & 0 & 12388.6 & 12388.6 \\
\hline & $\begin{array}{l}\text { Low } \\
\text { cost }\end{array}$ & $c_{r}=15$ & 167.5 & 120.833 & 80.78 & 58.5 & 35.5 & 94 & 275.8 & 12402.5 & 12678.3 \\
\hline
\end{tabular}

We plot the price and demand as a function of the cross-price sensitivity coefficients $(\beta)$, as illustrated in Figure 1 and Figure 2 when retailer is in a high cost state. We also plot the price and demand as a function of $\beta$, as shown in Figure 3 and Figure 4 when retailer's cost is in a low state. Fig.1 and Fig.3 show that the prices always increase with $\beta$ increasing for both a high cost state and a low cost state, and a wholesale price lower than the direct sale price, which prevents the retailer from obtaining products from the direct channel.

Fig.2 and Fig.4 show that the demand for both channels always increases with increasing $\beta$ and the retail sale demand is larger than direct sale demand. $\beta$ reflects the degree of 
competition and migration rate between two channels. The higher is $\beta$, the higher is the competition degree between the two channels, the demands and prices increase for both channels and both the manufacturer and the retailer enjoy greater profits. The managerial guideline we obtain from here is that the manager should increase $\beta$ by channel collaboration and post-sales services (Chen et al., 2012).

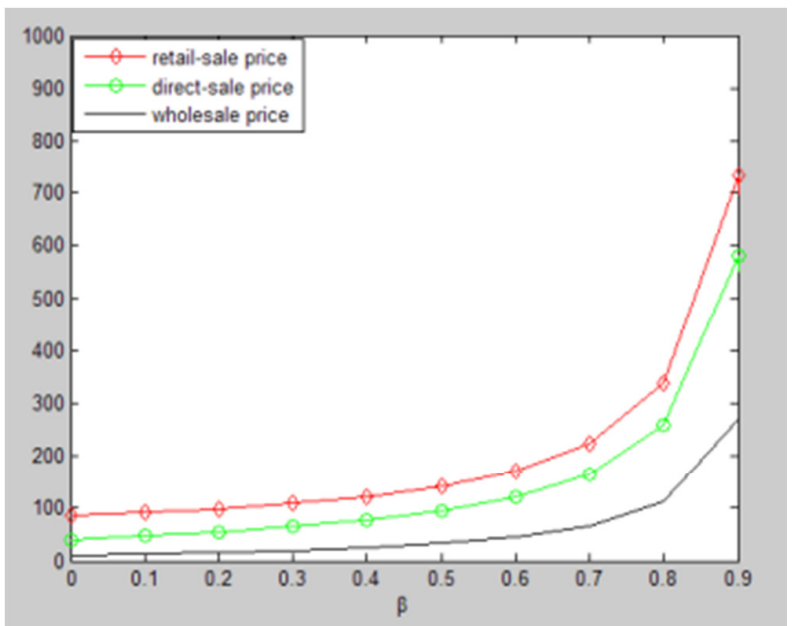

Fig.1 The change trend of prices with ${ }^{\beta}$ under asymmetric information when the retailer's cost state is high.

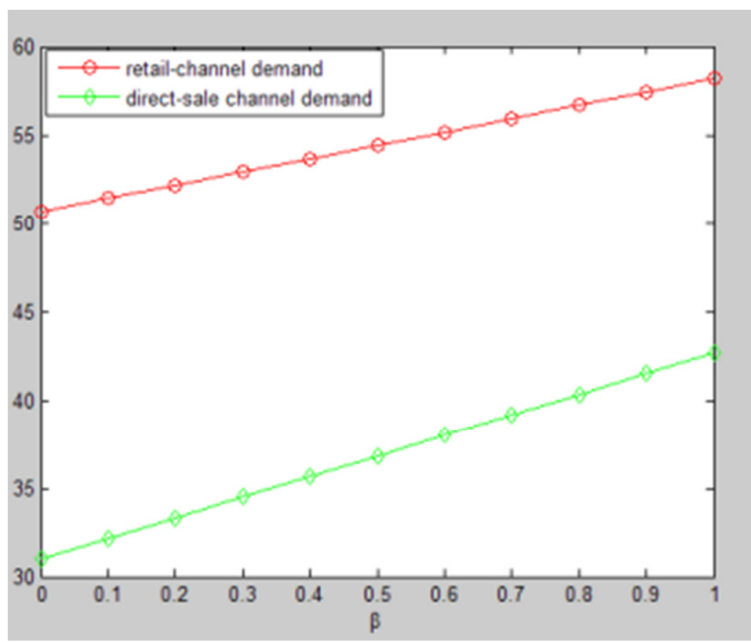

Fig.2 The change trend of demands with ${ }^{\beta}$ under asymmetric information when the retailer's cost state is high. 


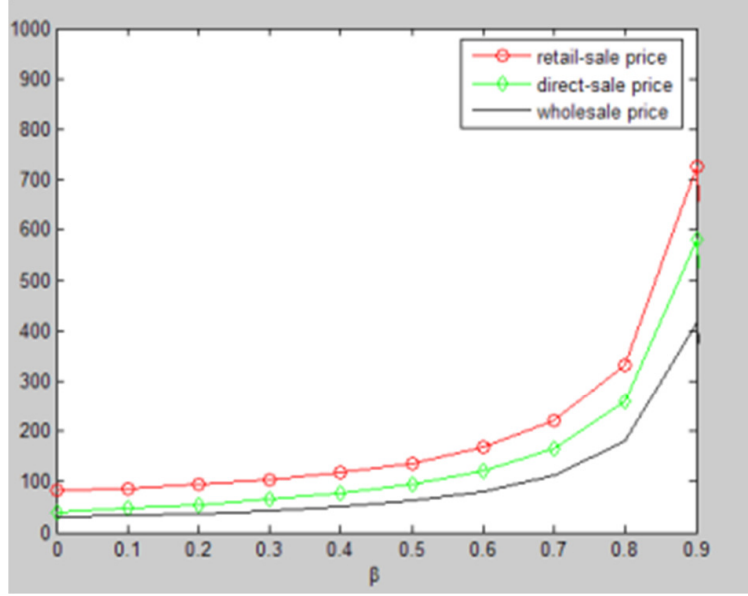

Fig.3 The change trend of prices with ${ }^{\beta}$ under asymmetric information when the retailer's cost state is low.

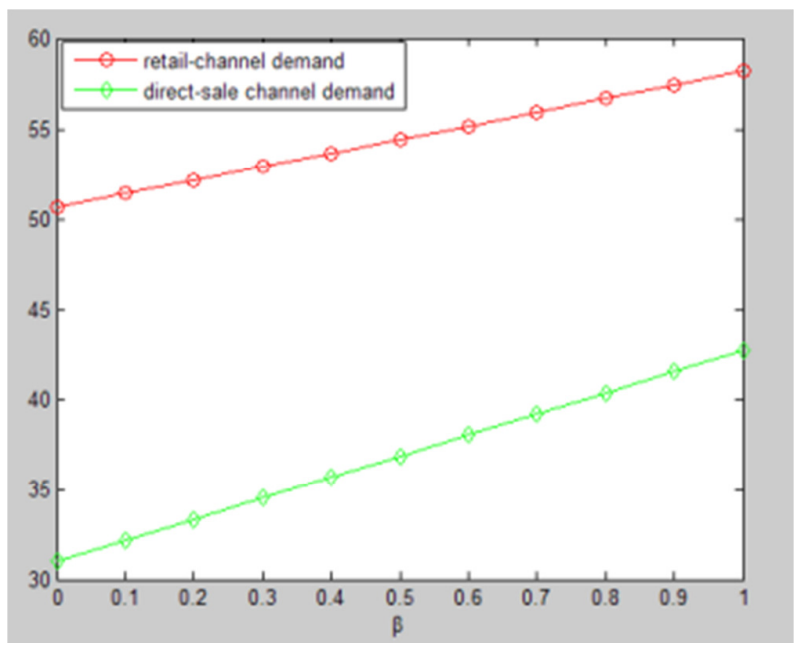

Fig.4 The change trend of demand with ${ }^{\beta}$ under asymmetric information when the retailer's cost state is low

We also plot the profit difference as functions of $\beta$ and $\rho$ as shown in Figure 5 and Figure 6 respectively. Fig. 5 shows that the amount of profit increase for the manufacturer from asymmetric information to full information increased with $\beta$ is increasing. The amount of profit decrement for the retailer from asymmetric information to full information decreased with $\beta$ is increasing. It can be seen that in Fig.5 the manufacturer benefits from sharing information at the cost of retailer's profit loss. With $\beta$ increasing, the higher is the competition degree between two channels, more profitable in dual-channel supply chain by sharing information than asymmetric information. 


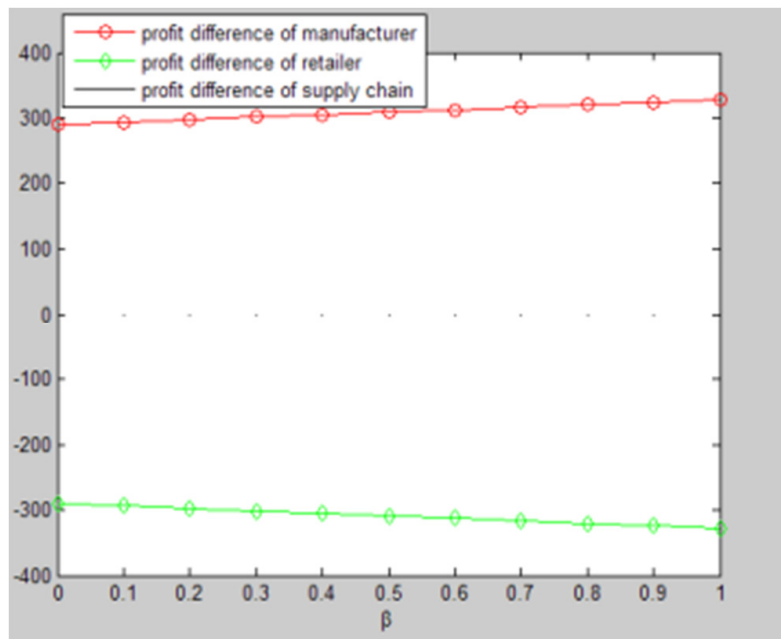

\section{Fig.5 The change trend of profit differences with ${ }^{\beta}$ when retailer's cost state is low}

From Section 3, we know that $\rho$ and $(1-\rho)$ respectively represent the end customer's preference for the direct channel and retail sale channel. From Fig. 6, we find that the profit difference for the manufacturer decreases with increasing $\rho$ from full information case to the asymmetric information case, while the profit difference increases for the retailer with increasing $\rho$ due to a negative profit difference from the full information case to the asymmetric information case. An important managerial insight obtained here is that the manufacturer can obtain a greater profit increment by sharing the retailer's cost information when the end customer prefers to buy its products from a retail sale channel $(1-\rho)$. The higher is the end customer's preference $(1-\rho)$ for the retail sale channel, the greater is the value of information sharing for the retailer and the greater the profits the retailer obtains from information asymmetry. Therefore, the manufacturer as a leader in a dual-channel supply chain should expand its market share of the direct channel and cultivate faithful customers that can effectively compensate any manufacturer's reduction in profit due to asymmetric information. 


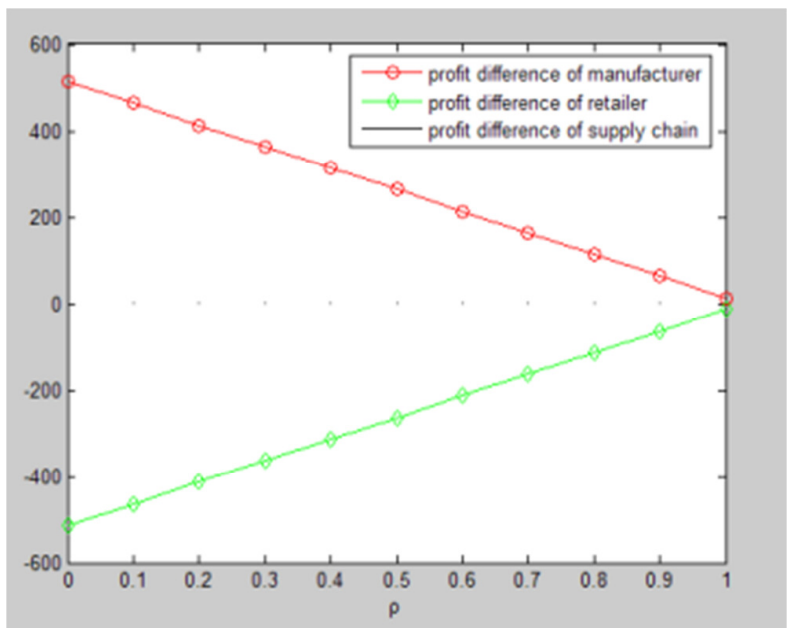

Fig.6 The change trend of profit difference with $\rho$ when retailer's cost state is low

\section{Conclusions}

Information asymmetry affects the decisions of the involved partners' and always results in reduced performance in a traditional retail supply chain. This paper studies the optimal contract design problem in a dual-channel supply chain that consists of traditional retail channel and a direct sale channel. We examine a situation where the retailer's cost is either high state or low state and where this information is private. A model has been developed to determine the manufacturer's optimal decisions as a contract design problem. The closed-form expressions are derived under both the asymmetric information case and the full information case. In addition, the optimum decisions are contrasted between the asymmetric information case and the full information case. We analyse the effect of information asymmetry on the profits of each channel partner and quantify the value of information sharing. This study finds that information asymmetry imposes inefficiency on the manufacturer and the whole supply chain, while the retailer benefits from asymmetric information. Our findings show that, in the Internet era, it is beneficial to the manufacturer to actively pursue access to the retailer's private information by way of offering the retailer an incentive mechanism share this private information. The retailer may share its private information if the lump sum side payment that the manufacturer offers can make up any reduction in the retailer's profit. 
This paper can be extended in several different directions, such as extending information asymmetry to a generalized case with continuous states rather than two-states as in our model. It may also be useful to consider some other contract menus and other kinds of demand function rather than the linear type adopted in our model. Another meaningful direction is to consider a dual-channel supply chain with multiple manufacturers or retailers under asymmetric information.

\section{References}

Cachon, G.P. and Lariviere, M.A. 2005. 'Supply Chain Coordination with Revenue-Sharing Contracts: Strength and Limitations'. Management Science, 51(1), 30-44.

Cachon, G. 2003. 'Supply Chain Coordinated with Contracts', in Handbooks in Operations Research and Management Science: Supply Chain Management, edited by Steve Graves and Tonde Kok, North Holland.

Cai, Gangshu (George) 2010. 'Channel Selection and Coordination in Dual-Channel Supply Chains'. Journal of Retailing, 86 (1), 22-36.

Cakanyildirim, M., Feng, Q., Gan, H., Sethi, P. 2012. 'Contracting and Coordination under Asymmetric Production Cost Information'. Production and Operations Management, 21 (2), 345-360.

Charles, C., Deming, Z., Christopher, T. 2004. 'Designing Supply Contracts: Contract Type and Information Asymmetry'. Management Science, 50(4), 550-559.

Chen, J., Zhang, H., Sun, Y. 2012. 'Implementing Coordination Contracts in a Manufacturer Stackelberg Dual-Channel Supply Chain'. Omega, 40(5), 571-583.

Chiang, K., Chhajed D., Hess, D. 2003. 'Direct Marketing, Indirect Profits: A Strategic Analysis of Dual-Channel Supply Chain Design'. Management Science, 49(1), 1-20.

Dan, B., Xu, G., Liu, C. 2012. 'Pricing Policies in a Dual-Channel Supply Chain with Retail Services'. International Journal of Production Economics, 39(1), 312-320.

Dumrongsiri, A., Fan, M., Jain, A., Moinzadeh, K. 2008. 'A Supply Chain Model with Direct and Retail Channels'. European Journal of Operational Research, 187(3), 691-718.

Hua, G., Wang, S., Cheng, T.C.E. 2010. 'Price and Lead Time Decisions in Dual-Channel 
Supply Chains'. European Journal of Operational Research, 205(1), 113-126.

Huang, S., Yang, C., Zhang, X. 2012. 'Pricing and Production Decisions in Dual-Channel Supply Chains with Demand Disruptions'. Computers \& Industrial Engineering, 62, 70 83.

Laffont, J., Martimor, D. 2002. The Theory of Incentives: The Principal-Agent Model. Princeton University Press, Princeton.

Lei Dong, Li Jianbin, Liu Zhixue. 2012. 'Supply Chain Contracts under Demand and Cost Disruptions with Asymmetric Information'. International Journal of Production Economics, 139(1), 116-126.

Li Sijie, Hua Zhongsheng. 2008. 'A Note on Channel Performance under Consignment Contract with Revenue Sharing'. European Journal of Operational Research, 184, $793-$ 796.

Samar, M., Dongqing, Y., Xiaohang, Y. 2008. 'Information Sharing of Value-Adding Retailer in a Mixed Channel Hi-Tech Supply Chain'. Journal of Business Research, 61(9), 950958.

Swaminathan, J., Tayur, S. 2003. Models for Supply Chains in E-Business. Management Science 49, 1387-406.

Volodymyr, B., Hantao, L., Peter, R., Yunzeng, W. 2012. 'Contracting with Asymmetric Demand Information in Supply Chains'. European Journal of Operational Research, 217, 333-341.

Wang Y., Jiang L., Shen Z. 2004. 'Channel Performance under Consignment Contract with Revenue Sharing'. Management Science, 50(1), 34-47.

Yan, R. 2008. 'Pricing Strategy for Companies with Mixed Online and Traditional Retailing Distribution Markets'. Journal of Production and Brand Management, 17, 48-56.

Yao Z., Stephen C.H. Leung, K.K. Lai. 2008. 'Manufacturer's Revenue-Sharing Contract and Retail Competition'. European Journal of Operational Research, 186, 637-651.

Yao, D., Liu, J. 2003. 'Channel Redistribution with Direct-Selling'. European Journal of Operational Research, 144(3), 646-658. 